\title{
A fault diagnosis approach for railway track circuits trimming capacitors using EMD and Teager Energy Operator
}

\author{
S. P. Sun ${ }^{1}$, H. B. Zhao ${ }^{2}$ \& G. Zhou ${ }^{1}$ \\ ${ }^{I}$ State Key Laboratory of Rail Traffic Control and Safety, \\ Beijing Jiaotong University, China \\ ${ }^{2}$ School of Electronic and Information Engineering, \\ Beijing Jiaotong University, China
}

\begin{abstract}
The trimming capacitor is an important component of a track circuit used for train detection in the railway train control system. In order to ensure the required dependability and availability levels, their working conditions shall be monitored in a timely and efficient manner. This paper presents a diagnosis approach of trimming capacitors based on Empirical Mode Decomposition (EMD) and Teager Energy Operator (TEO) theory. The EMD is used to decompose the short-circuit current signal into two parts. One is the sum of a set of Intrinsic Mode Functions (IMFs), which feature the fault information of the defective trimming capacitors; the other is the residual element relating to the signal variation trend. Then the TEO of IMFs is performed and the instantaneous frequencies are derived, so as to highlight the fault features. Experiments with simulated data show that multiple defects can be detected effectively using this approach. Compared with the existing methods which are only able to detect one failed capacitor, the integrated method represents a rather improved performance.

Keywords: track circuit, trimming capacitor, Empirical Mode Decomposition, Teager Energy Operator.
\end{abstract}

\section{Introduction}

A railway track circuit is used for train detection as well as track-to-train information transmission of the automatic train control system in China. The 
trimming capacitor is an essential component of a track circuit and its main function is to compensate the transmission loss of the track circuit signal. Trimming capacitors are connected between the two rails with constant spacing along the track. According to on-site research, trimming capacitors are often removed due to aging, vibrations of the train running behaviour, atmospheric conditions or track maintenance operations. It has become a common fault of a railway track circuit. Experiments show that a track circuit could go to wrong operation if there exist three defective trimming capacitors, even one is removed at adverse condition (Zheng [1]). Trimming capacitors are inspected by inspection vehicles with a scheduled maintenance regime (Zhao et al. [2]). This type of maintenance scheme is costly and time-consuming. In order to reduce maintenance costs and provide a high level of reliability and safety, a great deal of research effort has been directed towards fault detection and diagnosis for trimming capacitors in recent years. One important direction is through analysing the so called short-circuit current signal, and excellent progress has been made using the Dempster-Shafer theory (Oukhellou et al. [3]), discrete binary wavelet transform (Zhao et al. [4]), partial least squares regression and neural network (Debiolles et al. [5]). But most of these methods are only able to detect the first one defective trimming capacitor. The situation of multiple concurrent defects is not being examined.

In this paper, we explore the use of an integrated approach of EMD and TEO theory to diagnose trimming capacitors. At first, the basic principle of a track circuit is described and the simulated short-circuit current signal is obtained from its electric model. Then EMD and TEO are outlined for their application. The results of this method with simulated data are present and analysed in section 4 . At last, section 5 draws our conclusions with discussion and further work.

\section{Track circuit principle}

A track circuit is a safety feature designed to detect the presence of trains on a railroad track. It is a complex system consisting of the following components:

- A transmitter is used to generate the suitable coded data which contains running information such as authorized speed.

- The two rails are used for a transmission line of the track circuit signal.

- Two electrical separation joints are used to separate the signals from neighbour track circuits.

- Trimming capacitors connected between the two rails at constant spacing are used to compensate the transmission loss of track circuit signal because of the inductive behaviour of the track.

- A receiver, which is located to the opposite end from the transmitter, detects the signal from the transmitter. It is connected to a relay used to report whether or not the track is occupied.

When a track circuit is unoccupied by a train (fig. 1), the coded data will be transmitted to the receiver via the two running rails, and its detection energizes a 
track relay. If a train is present (fig. 2), it creates a short circuit in the track circuit, so the transmitted signal does not reach the receiver and the track relay is not energized. In China railway domain, the track circuit is also used to transmit running information to the train from the rails. The sensor equipped in front of the first axle picks up the transmitted information, i.e. short-circuit current $\left(I_{c c}\right)$ signal when a track circuit is shunted by a train.

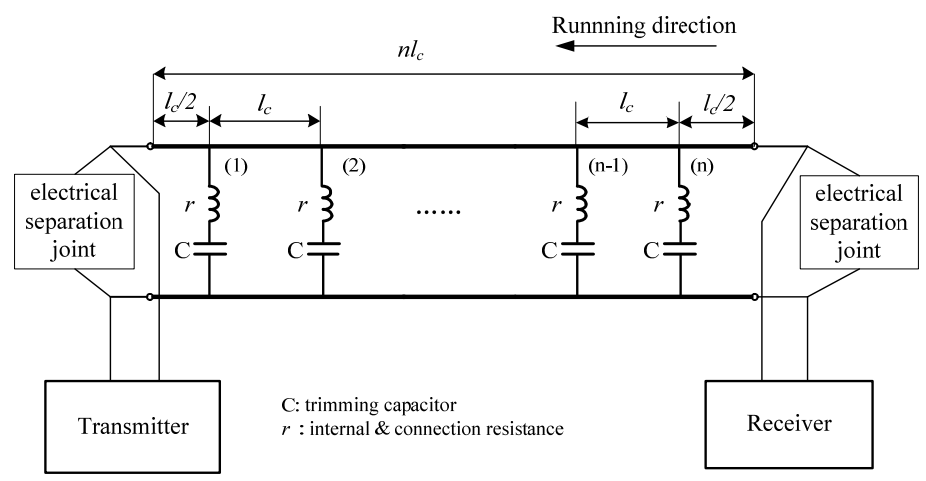

Figure 1: Track circuit mode-track unoccupied.

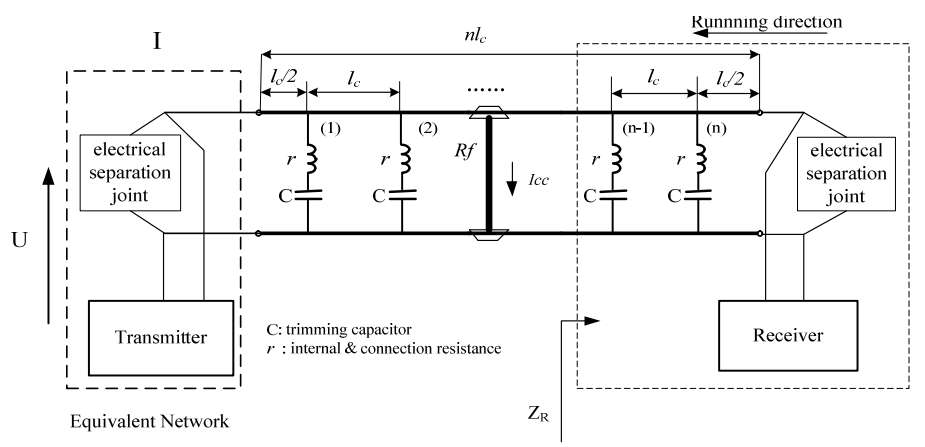

Figure 2: Track circuit mode-track occupied.

An electrical model is developed that can be able to perform realistic simulations of short-circuit current using transmission line theory (Fessant $e t$ al. [6]). As shown in fig. 2, when the track circuit is shunted, the first wheelset can be seen as a resistance called shunt resistance $R_{f}, R_{f} \in[0.06 \Omega, 0.15 \Omega]$. $Z_{R}$ denotes the equivalent impedance seen from the shunt point to the receiver, $R_{f}<<Z_{R} . I_{c c}$ can be calculated by eqn (1):

$$
\left(\begin{array}{l}
U \\
I
\end{array}\right)=Q \cdot\left(\begin{array}{l}
I_{c c} R_{f} \\
I_{c c}
\end{array}\right)
$$


So

$$
I_{c c}=\frac{U}{Q_{11} R_{f}+Q_{12}}
$$

$Q$ denotes the transmission matrix from transmitter to shunt point; it is a cascade of the rail and the trimming capacitor transmission matrixes. Fig. 3 shows the comparison of the simulated and real $I_{c c}$ signal of a track circuit. The signals are normalized.

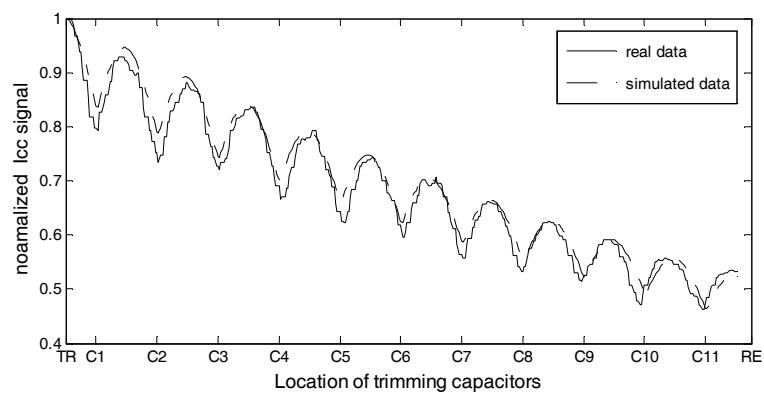

Figure 3: $\quad$ Comparison of simulated and real $I_{c c}$ signal.

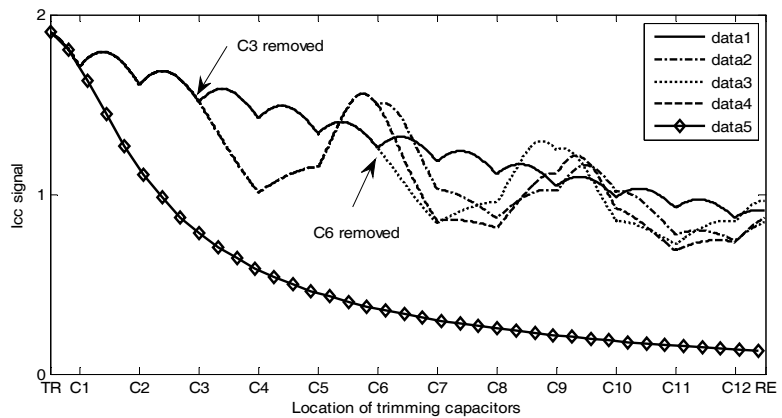

Figure 4: $\quad$ Examples of simulated $I_{c c}$ signal.

Fig. 4 shows examples of $I_{c c}$ signals simulated along a $960 \mathrm{~m}$ long track circuit with 12 trimming capacitors, and the spacing between two capacitors is $80 \mathrm{~m}$. The signals are measured with 4 points per meter. The following observations can be found:

- The curve between two trimming capacitors exists in the form of an arch. The minima point of each arch is correspond to a trimming capacitor, and the defective capacitor only affects the shape of the arches between the fault and the receiver.

- The curve of the $I_{c c}$ signal is equivalent to the absolute value of a amplitude modulation signal in the exponential form (Zhao and $\mathrm{Mu}[7]$ ). 
- $\quad$ There exists a down peak (high frequency) at each trimming capacitor location while at other positions the signal depicts a slow change (low frequency). If a trimming capacitor is removed, the corresponding peak disappears.

\section{Fault detection and diagnosis methodology}

\subsection{Overview of the diagnosis approach}

The aim of the fault diagnosis approach is to detect the removed capacitors by analysing the $I_{c c}$ signal based on the above three observations. This method consists in decomposing $I_{c c}$ by EMD to obtain the sum of IMFs, i.e. ( $\left.\sum i m f_{i}\right)$, then calculating the instantaneous frequencies of the $\left(\sum i m f_{i}\right)$ by TEO. A decision regarding to whether defects exist is made according to the modifications of the instantaneous frequencies.

\subsection{EMD description}

EMD is a key part of Hilbert-Huang Transform (HHT). This method can decompose any complicated data set into a finite and often small number of IMFs that admit well-behaved Hilbert transforms. It is applicable to nonlinear and non-stationary processes (Huang et al. [8]). The IMFs satisfy the two requirements:

- $\quad$ The number of extrema and the number of zero crossings in the IMF must either be equal or differ at most by one;

- At any point the mean value of the envelopes defined by the local maxima and local minima must be zero.

The EMD algorithm can be implemented by the sifting process including the following steps:

Step1: Identify all the local maxima of the original input signal $x(t)$, then fit all the local maxima by a cubic spline line interpolation for use as the upper envelope $X_{u}(t)$. Then perform the same operation to the local minima to produce the lower envelope $X_{L}(t)$.

Step2: Calculate the mean envelop of $X_{u}$ and $X_{L}$, and it is defined as $m_{l}$.

Step3: Define the first component as:

$$
x(t)-m_{1}=h_{1}
$$

Step4: If $h_{l}$ is not an IMF, treat $h_{l}$ as new original data and repeat the previous process. Then set

$$
h_{1}-m_{11}=h_{11}
$$

Repeat this sifting procedure $k$ times until $h_{l k}$ is an IMF; that is

$$
h_{1(k-1)}-m_{1 k}=h_{1 k}
$$


Then designate eqn (6)

$$
c_{1}=h_{1 k}
$$

as the first IMF component of original signal.

Step5: Calculate the residual

$$
r_{1}=x(t)-c_{1}
$$

If $r_{l}$ has less than 2 minima or 2 extrema, the extraction is finished. Else treat $r_{l}$ as the new data and subject to the same sifting process as described above.

After this decomposition the original signal $x(t)$ can be expressed as eqn (8)

$$
x(t)=\sum_{i=1}^{n} c_{i}+r_{n}
$$

$c_{i}$ denotes the $i^{\text {th }}$ empirical mode, and $r_{n}$ denotes the residual relating to signal trend. This decomposition structure can be explained as fig. 5 .

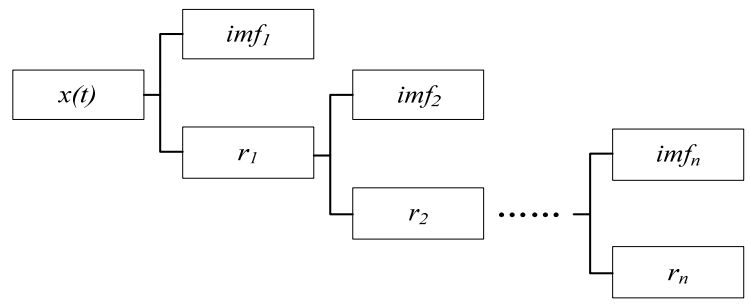

Figure 5: $\quad$ EMD decomposition structure.

\subsection{TEO description}

The TEO, proposed by Teager and Kaiser, is a non-liner operator. It is a useful tool for time-frequency analysis and has been widely used in a number of applications such as signal /image processing, fault diagnosis and so on. The TEO has a small time window, making it ideal for local (time) analysis of signals (Teager and Teager [9], Maragos et al. [10]).

In the continuous domain the TEO is defined as

$$
\psi[x(t)] \triangleq\left(\frac{d x(t)}{d t}\right)^{2}-x(t) \frac{d^{2} x(t)}{d t^{2}}
$$

and as

$$
\psi[x(n)] \triangleq x^{2}(n)-x(n-1) x(n+1)
$$

in the discrete case.

For a discrete-time real-valued amplitude-modulation-frequency-modulation signal the instantaneous frequency can be estimated by eqn (11) 


$$
\Omega(n) \approx \arccos \left(1-\frac{\psi[x(n)-x(n-1)]}{2 \psi[x(n)]}\right)
$$

Compared to HHT, The implementation of TEO needs only three sampling points every time without complex calculation, the time-consuming is lower.

\section{Application of the approach to case illustration}

The diagnosis approach starts with a decomposition of the $I_{c c}$ signal using EMD. With the simulated $I_{c c}$ signal without defective capacitors of the fig. 4, the results of EMD process are as shown in fig. 6. White Gaussian noise is added to the original signal, the percentage of the standard deviation is $5 \%$.
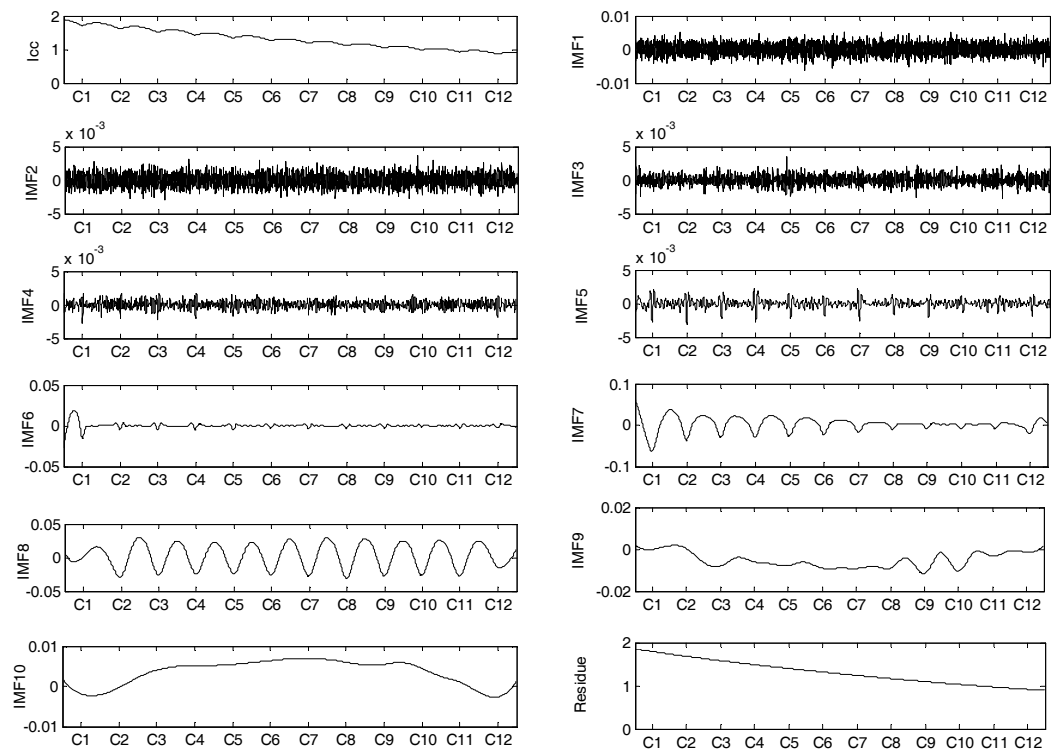

Location of trimming capacitors

Figure 6: Decomposition of a simulated signal without defect.

Fig. 7 shows that the $\left(\operatorname{\sum imf}_{i}\right)$ and its instantaneous frequencies calculated by TEO with the simulated signal of fig. 6 . The same processes are applied to the signals for three working states of a track circuit: one removed capacitor at the position of $\mathrm{C} 6$, two removed capacitors at $\mathrm{C} 3, \mathrm{C} 6$ and three removed capacitors at $\mathrm{C} 2, \mathrm{C} 8, \mathrm{C} 11$. The results are presented in figs. 8, 9 and 10, respectively. The instantaneous frequencies are normalized. 

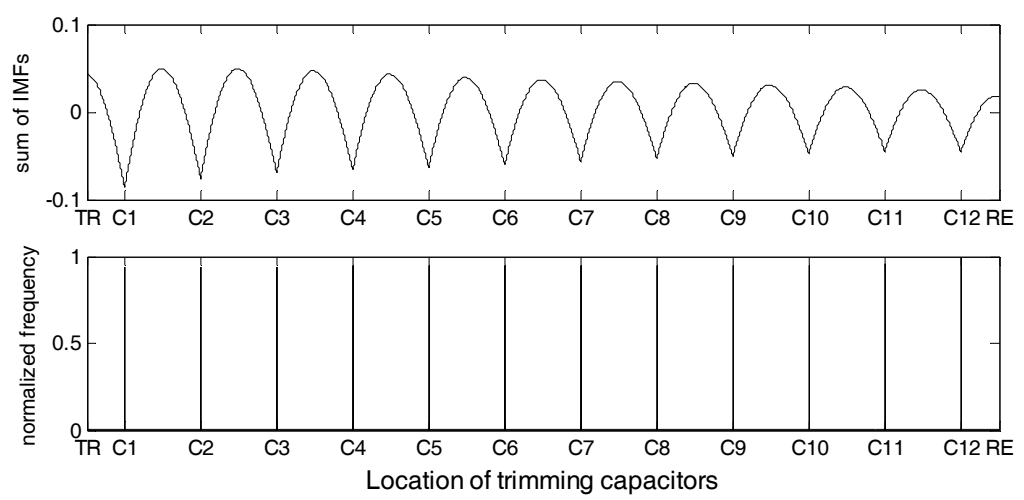

Figure 7: $\quad \operatorname{Limf}_{i}$ and instantaneous frequencies without defect.
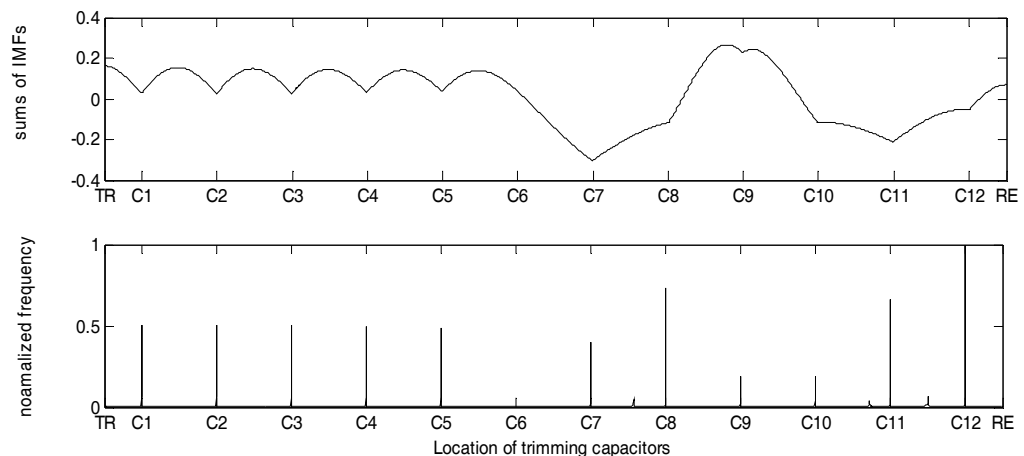

Figure 8: $\quad \operatorname{Limf}_{i}$ and instantaneous frequencies with one removed trimming capacitor.
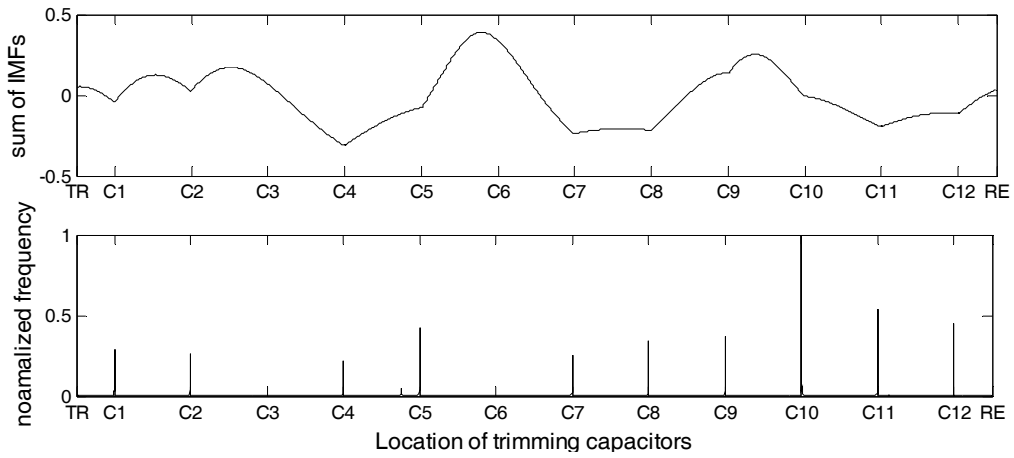

Figure 9: $\quad \operatorname{Limf}_{i}$ and instantaneous frequencies with two removed trimming capacitors. 

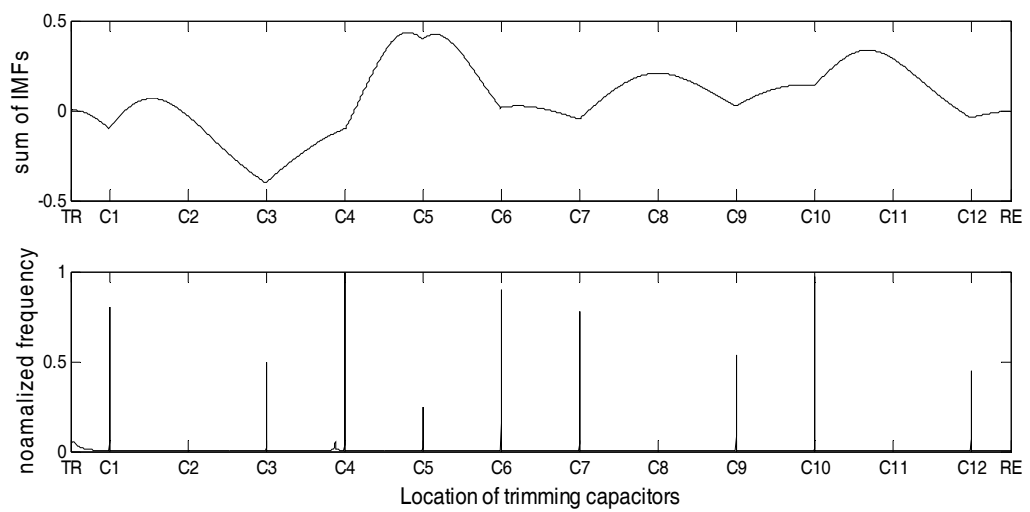

Figure 10: $\quad \operatorname{Limf}_{i}$ and instantaneous frequencies with three removed trimming capacitors.

We can see that, the instantaneous frequencies are affected principally by the removed trimming capacitors, and the defects can be diagnosed by the modifications of the instantaneous frequencies. According to the adjustment sheets of the track circuits (Ministry of Railway China [11]), the simulated data are obtained at different situations in terms of the length of a track circuit, the number of trimming capacitors, the carrier frequency and the ballast resistance. Experiments with all these data show that the defects can be well detected by the proposed approach while the threshold is set to ten per cent of the maximum instantaneous frequency. This performance represents an improvement as compared with the state of reference methods.

\section{Conclusion}

This paper does an exploratory research on the fault diagnosis for the railway track circuit trimming capacitors. A novel diagnostic system has been developed based on the combined use of EMD and TEO theory. Experiments show that all defective trimming capacitors are detected and no false alarms are produced. Multiple concurrent defective trimming capacitors can be examined by this approach, which represents an improvement over the state of the art reference methods. The adaptability of this approach is tested thoroughly by the changes experienced on the track circuits. The result is conclusive although no results with real data could be presented. Further work is underway to verify the system in the long term, using operational data sets from the cab signalling on-board system already in service on railways in China. 


\section{Acknowledgement}

This work was financially supported by The High-tech R\&D project of The Ministry of Railways of the People's Republic of China (Grant No. 2011X021c). The authors acknowledge this support.

\section{References}

[1] Zheng F.L., Analysis and discussion on reason and protection to failure of compensation capacitor. Railway Computer Application, 18(11), pp. 52-53, 2009.

[2] Zhao H.B., Liu Y. and Liu B., Research on the onboard auto-test system for track circuit compensating capacitors. 10th International Conference on Computer System Design and Operation in the Railway and Other Transit Systems, eds. J. Allan, C.A. Brebbia and A.F. Rumsey, WITPress: Prague, pp. 965-971, 2006.

[3] Oukhellou L., Debiolles A., Denoeux T. and Aknin. P., Fault diagnosis in railway track circuits using Dempster-Shafer classifier fusion. Engineering Applications of Artificial Intelligence, 23(1), pp. 117-128, 2010.

[4] Zhao L.H, Li Z., and Liu W., The compensation capacitors fault detection method of jointless track circuit based on DBWT and WR. IEEE International Conference on Intelligent Computing and Intelligent Systems, IEEE Computer Society: Shanghai, pp. 875-879, 2009.

[5] Debiolles A, Oukhellou L and Aknin P. Combined use of partial least squares regression and neural network for diagnosis tasks. Proceedings of the 17th International Conference on Pattern Recognition, IEEE Computer Society: Cambridge, pp. 573-576, 2004.

[6] Fessant F., Aknin P., Vilette F. and Antoni, M., Modélisation électrique du circuit de voie, élément du système de transmission voie-machine des TGV. Revue 3EI, 27, pp. 46-52, 2001.

[7] Zhao L.H. and Mu J.C., Fault diagnosis method for jointless track circuit based on AOK-TFR. Journal of Southwest Jiaotong University. 46(1), pp. 84-91, 2011.

[8] Huang N., Shen Z., Long S., et al, The empirical mode decomposition and the Hilbert spectrum for nonlinear and non-stationary time series analysis. Proceedings:Mathematical, Physical and Engineering Sciences, 454(1971), pp. 903-995, 1998.

[9] Teager H.M. and Teager S.M., Evidence for nonlinear sound production mechanisms in the vocal tract. NATO Advanced Study Institute: Boston, pp. 241-261, 1990.

[10] Maragos P, Kaiser J.F. and Quatieri T.F., Energy separation in signal modulations with application to speech analysis. Signal Processing, 41(10), pp. 3024-3051, 1993.

[11] Ministry of Railway China, The Technical Standards of Railway Signaling MaintenanceII, China Railway Publishing House: Beijing, pp. 375-425, 2006. 